\title{
RECEITAS E GASTOS GOVERNAMENTAIS: UMA ANÁLISE DE CAUSALIDADE PARA O CASO BRASILEIRO
}

\author{
Cleomar Gomes da Silva* \\ SÉrgio Jurandyr Machado ${ }^{\dagger}$ \\ Denílson Torcate Lopes $\ddagger$ \\ André Marques Rebelo $§ \mathbb{I}$
}

\begin{abstract}
Resumo
O objetivo deste artigo é analisar o debate entre arrecadação e gastos públicos governamentais para o caso brasileiro, isto é, se a despesa pública é a responsável por gerar constantes aumentos da arrecadação governamental brasileira, ou se a causalidade é reversa. A metodologia econométrica utilizada envolve técnicas de cointegração e de modelo de correção de erros para o período compreendido entre o 1 o trimestre de 1999 e o 3 o trimestre de 2008. Os resultados econométricos mostram que, no período analisado, o Estado brasileiro pode ser caracterizado pela utilização de um modelo fiscal do tipo "gastar e arrecadar". Isso significa que elevações nos dispêndios governamentais provocam mudanças permanentes na arrecadação pública.
\end{abstract}

Palavras-chave: Política Fiscal, Gasto Público, Análise de Séries Temporais

JEL classification: C32, E62, H6

\begin{abstract}
The aim of this paper is to analyze the tax-spend debate for the Brazilian case, that is, if an increase in public spending leads to an increase in government revenues, or if there is a reverse causality (the increase in government revenues leading to an increase in public spending). The empirical analysis is based on cointegration and vector error correction models and the data ranges from the 1st quarter/1999 to the 3rd quarter/ 2008. The results show that the Brazilian government follows a "spend and tax" policy. It means that more government spending leads to permanently higher taxes.
\end{abstract}

Palavras-chave: Fiscal Policy, Public Spending, Time Series Models JEL classification: C32, E62, H6

\footnotetext{
* Instituto de Economia - Universidade Federal de Uberlândia. E-mail:cleomargomes@ie.ufu.br

${ }^{\dagger}$ Insper Instituto de Ensino e Pesquisa. E-mail: SergioJM@insper.org.br.

‡ FIESP (Departament of Economics). E-mail: denilson.lopes@fiesp.org.br.

$\S$ FIESP (Departament of Economics). E-mail: arebelo@fiesp.org.br.

II Este artigo é uma versão modificada e atualizada do artigo "Arrecadar e Gastar ou Gastar e Arrecadar? Evidências para o Caso Brasileiro", apresentado no 36 Encontro Nacional de Economia - ANPEC 2008. Os autores agradecem os comentários dos pareceristas anônimos.
} 


\section{Introdução}

Uma questão que tem suscitado debates constantes no Brasil está relacionada às dinâmicas do gasto público e da arrecadação tributária no país. Uma rápida análise dos resultados fiscais brasileiros mostra aumentos consideráveis da carga tributária e do gasto público governamental. Dados oficiais deixam claro que a carga tributária como proporção do PIB cresceu cerca de 11 pontos percentuais entre 1990 e 2008 . No mesmo sentido, o crescimento real da receita líquida governamental passou de 5,3\% em 2000 para 9,8\% em 2007 e $7,6 \%$ em 2008, ao passo que o crescimento real da despesa passou de $6,3 \%$, em 2000, para 9,3\%, em 2007 e 3,4\% em $2008^{1}$.

Isso tem levantado preocupações e perguntas em torno da melhor forma de frear esta dinâmica perversa que tem como uma das consequências principais o entrave do crescimento econômico brasileiro. Em outras palavras, a compreensão mais clara da relação intertemporal entre as receitas e os dispêndios governamentais torna-se essencial para projetos de correção de desequilíbrios fiscais no Brasil ${ }^{2}$.

Neste sentido, um debate importante está relacionado à causalidade do processo, ou seja, a pergunta a ser feita é se um aumento da carga tributária carrega consigo um aumento do gasto público, ou se o processo ocorre na direção contrária. É justamente esta causalidade existente entre arrecadação e gasto governamental no Brasil o objetivo deste trabalho. O período de análise está compreendido entre o primeiro trimestre de 1999 e o terceiro trimestre de 2008 e a metodologia econométrica utilizada é a análise de cointegração conjugada com o modelo de correção de erros. O artigo contribui para a literatura aplicada ao caso brasileiro na medida em que dá enfoque ao período recente da história fiscal brasileira, superando o problema de dados inerente ao período anterior ao ano de 1999. Os resultados econométricos mostram que, no período analisado, o Estado brasileiro pode ser caracterizado pela utilização de um modelo fiscal do tipo "gastar e arrecadar".

O restante do artigo está organizado da seguinte maneira. A seção 2 discute as hipóteses e a literatura relacionadas ao tema. A seção 3 descreve a metodologia econométrica e os dados. A seção 4 reporta os resultados e a última seção faz a conclusão do trabalho.

\section{Hipóteses e análise da literatura}

Há vários modelos que objetivam caracterizar a relação intertemporal entre receitas e despesas governamentais. Primeiramente, a hipótese "arrecadar e gastar" (Friedman 1967, Barro 2003, Becker et al. 2003) parte do princípio

\footnotetext{
${ }^{1}$ O crescimento real da despesa no ano de 2008, embora ainda significativo, pode ser considerado atípico por dois motivos: primeiro, a negociação por aumentos salariais do funcionalismo público se estendeu por vários meses, o que postergou parte significativa do impacto associado aos aumentos para 2009. Adicionalmente, o nível de atividade econômica (especialmente nos 3 primeiros trimestres) viabilizou a redução temporária de uma série de gastos associados às operações oficiais de crédito e a subsídios e subvenções econômicas.

${ }^{2}$ Certamente, o ideal seria uma abordagem dos gastos do governo desagregados em gastos chamados de produtivos e gastos chamados de improdutivos, tal como discutido em Barro \& SalaI-Martin (1990), Devarajan et al. (1996). No entanto, esta forma de cálculo se torna inviável para o caso brasileiro uma vez que os dados de investimentos governamentais fornecidos pelo Tesouro Nacional não estão disponíveis para todo o período de análise. Além disso, gastos relacionados como "improdutivos" (como Universidades Federais) podem ser classificados de outra forma.
} 
de que mudanças na receita governamental provocam alterações nos gastos públicos. Isso significa que a relação causal seria de uma elevação da arrecadação do governo para um consequente aumento de seus dispêndios. Desta maneira, esta literatura apregoa que uma redução de impostos implicaria em subsequentes cortes dos gastos governamentais, significando que o melhor a ser feito seria "starve the beast", isto é, deixar que a fera (o governo) passe fome para que o mesmo promova cortes de gastos públicos.

Por outro lado, a hipótese de um regime fiscal do tipo "gastar e arrecadar" apregoa uma relação reversa, ou seja, elevações temporárias nos dispêndios governamentais provocam mudanças permanentes na receita, via elevação da taxação (Chang \& Chiang 2009). Esta hipótese tem como defensores Peacock \& Wiseman (1961), que argumentam que aumentos temporários nas despesas públicas, devido a uma situação de crise fiscal, são geralmente utilizados para justificar maior carga tributária, que então se torna permanente.

Além destes dois argumentos, ainda existem as hipóteses do Sincronismo Fiscal e da Separação Institucional. Na primeira, tal como apregoado por Musgrave (1966), Meltzer \& Richard (1981), dentre outros, as decisões de receita e dispêndio são tomadas simultaneamente. Neste caso, os eleitores comparam os benefícios e custos marginais dos serviços públicos quando formulam decisões em termos de gastos e receitas públicas (Chang \& Chiang 2009). Já na Separação Institucional, as decisões sobre taxação são independentes da alocação das despesas governamentais. Isso acontece pela falta de consenso entre os tomadores de decisão do orçamento governamental, implicando em uma independência decisória entre receitas e despesas. Neste último caso, pode ser citado o trabalho de Baghestani \& McNown (1994), dentre outros.

\subsection{Revisão da literatura}

VonFurstenberg et al. (1986) utilizam a metodologia dos vetores auto-regressivos, para os dados trimestrais americanos no período 1954-1982. Os autores mostram que os gastos governamentais não respondem positivamente a choques de arrecadação, ou seja, a sequência "gastar e arrecadar" tem maior suporte empírico.

Hakkio \& Rush (1991) fazem hipóteses sobre o processo estocástico seguido pelas variáveis de gasto governamental e receita tributária e aplicam técnicas de cointegração como forma de testar a validade da restrição orçamentária intertemporal. Os autores afirmam que a condição necessária para que um governo obedeça a sua restrição intertemporal é a evidência de uma relação de longo prazo (de cointegração) entre o gasto governamental total, incluindo os juros da dívida pública e a arrecadação tributária. O raciocínio é que se ambas as séries forem não estacionárias, e se a restrição orçamentária intertemporal for válida, a cointegração faz com que a dívida pública descontada seja estacionária. Utilizando dados para a economia dos Estados Unidos, Hakkio \& Rush (1991) rejeitam a hipótese de ausência de cointegração entre gasto e arrecadação para o período 1950-1988.

Baghestani \& McNown (1994) encontram forte existência de uma relação de longo prazo entre receitas e despesas do governo norte-americano. Os autores chegam à conclusão de que o processo fiscal dos EUA pode ser caracterizado pela separação institucional entre as funções de alocação e taxação do governo, com respectivas elevações determinadas pelo crescimento econômico de longo prazo. 
Payne (1998) examina a relação temporal entre receitas e despesas para 48 estados norte-americanos entre os anos 1942 e 1992. Por intermédio dos modelos de correção de erros, o artigo dá suporte à hipótese "arrecadar e gastar" para 24 estados. A hipótese "gastar e arrecadar" é validada para 8 estados, enquanto a hipótese de sincronização fiscal é encontrada em 11 estados. Nos outros 5 estados não foi encontrado suporte para o modelo de correção de erros.

Darrat (1998) estuda a relação entre receitas e despesas para Turquia e acha um comportamento estável de longo prazo entre os gastos do governo e as receitas do país. O resultado encontrado também mostra que a dinâmica de curto prazo corrobora uma causalidade do tipo "arrecadar e gastar".

Cheng (1999) testa a causalidade entre impostos e gastos para 8 países da América Latina e encontra uma causalidade bi-direcional para Chile, Panamá e Peru. Para Colômbia, República Dominicana, Honduras e Paraguai, a causalidade encontrada vai da arrecadação para os gastos. Na opinião do autor, o estudo rejeita fortemente a hipótese "gastar e arrecadar".

Chang et al. (2002) também utilizam a metodologia de cointegração e de vetores auto-regressivos para testar as hipóteses de "arrecadar e gastar", "gastar e arrecadar" e sincronização fiscal para 10 países (Coreia, Taiwan, Tailândia, Austrália, Canadá, Japão, Nova Zelândia, África do Sul, Reino Unido, EUA). Os dados anuais compreendem os anos de 1951 a 1996 e os resultados dos autores mostram uma causalidade das receitas para os gastos, em favor da hipótese "arrecadar e gastar", para Japão, Coréia do Sul, Taiwan, Reino Unido e EUA. A relação oposta, a favor da hipótese "gastar e arrecadar", é encontrada para Austrália e África do Sul. O Canadá mostra uma causalidade dupla, em favor da sincronização fiscal. Já a Nova Zelândia e a Tailândia geram resultados que não corroboram nenhuma das hipóteses.

Romer \& Romer (2007) fazem uma ampla análise da hipótese "arrecadar e gastar" para o caso dos EUA, com foco no comportamento dos gastos como resultado de mudanças legislativas nos impostos que não foram motivadas por alterações planejadas nos gastos, por um desejo de reduzir o déficit orçamentário, ou por considerações macroeconômicas variadas. Os autores não encontraram resultados que comprovem a hipótese de que cortes nos impostos regulam os gastos do governo. No entanto, tais cortes podem causar um deslocamento da composição dos dispêndios em direção aos gastos com defesa. Os resultados também mostram que o principal efeito de modificações na estrutura da arrecadação é a tentativa governamental de provocar alterações na direção contrária, isto é, de novamente provocar aumentos de impostos.

Chang \& Chiang (2009) analisam gastos e receitas de 15 países da OCDE para o período 1992-2006, via utilização de dados de painel. O resultados do trabalho é de causalidade bi-direcional entre receitas e despesas, dando suporte à Hipótese de Sincronização Fiscal.

\subsection{Revisão da literatura referente ao caso brasileiro}

A grande maioria dos estudos sobre a sustentabilidade da dívida pública no Brasil, para o período anterior à reforma monetária de 1994, sugere que não somente a dívida é sustentável, mas que também o regime fiscal segue uma política do tipo "gastar e arrecadar". Isso significa que a decisão de política de gastos é tomada primeiramente, sendo então a arrecadação de impostos ajustada de maneira a equilibrar as contas do governo. 
Utilizando dados anuais das contas nacionais para o período 1947-1992, e seguindo o enfoque de Hakkio \& Rush (1991), Issler \& Piqueira (2000) sugerem que o orçamento do governo era equilibrado por meio de mudanças na receita, independentemente de qual era a origem do desequilíbrio, seja ele fruto de queda da receita ou de aumento dos gastos. O estudo de Issler \& Piqueira (2000) corrobora evidências anteriores de Pastore (1995) e Rocha (1997). Tais estudos mostram que a característica fundamental das finanças públicas era o uso da senhoriagem endógena de modo a acomodar o aumento exógeno dos gastos. Além da receita de senhoriagem, a subindexação da dívida, fruto da aceleração inflacionária que corroía parte da dívida herdada do passado, era outro elemento que impedia que a dívida assumisse uma trajetória explosiva, oferecendo-lhe um aspecto sustentável.

Baffes \& Shah (1994) testam a causalidade e o co-movimento de arrecadação e gastos para a Argentina, México e Brasil. Utilizando dados de 1908 a 1985, para o caso específico brasileiro, os autores acham válida a hipótese "arrecadar e gastar". Já para os casos argentino e mexicano, a hipótese de sincronismo fiscal se mostra mais coerente.

Mattos \& Rocha (2001) analisam o mecanismo orçamentário brasileiro e concluem que aumentos de gastos resultam em elevações de impostos, dando suporte à hipótese "gastar e arrecadar". Mello (2008) utiliza dados mensais de gastos e receita primária, para o período 1997-2005, e também encontra evidências de gastos exógenos no regime fiscal. Gamboa \& Silva (2004) utilizam séries temporais mensais, de julho de 1986 a outubro de 2003, e encontram evidências de um regime fiscal do tipo "gastar e arrecadar".

Por outro lado, Batolla (2004) utiliza dados trimestrais de receita (incluindo senhoriagem), despesa (incluindo pagamento de juros), de execução financeira do Tesouro e do PIB. Para o período 1992 a 2003, a autora encontra evidências de que o regime fiscal brasileiro segue um processo "arrecadar e gastar".

\section{Metodologia econométrica}

A análise empírica é baseada na metodologia econométrica de cointegração, cujo objetivo é evidenciar uma relação de equilíbrio entre variáveis econômicas ao longo do tempo. A ideia é que, embora as variáveis possam apresentar desvios em suas trajetórias de curto prazo, em virtude de choques que eventualmente acometem a economia, uma vez dissipados os efeitos de tais choques, estas variáveis convergem para um comportamento padrão sincronizado de longo prazo.

A avaliação da dinâmica do orçamento fiscal será feita por intermédio da abordagem de cointegração, tal como descrito em Johansen (1988). Com esta metodologia, obtêm-se evidências sobre a relação de longo prazo entre as variáveis estudadas. Entretanto, podem ocorrer desvios de curto prazo dessa relação de longo prazo, e esses desvios são captados pelo Modelo de Correção de Erros. Este não apenas indica a velocidade de ajuste dos desvios de curto prazo rumo ao equilíbrio de longo prazo, mas também, por meio da sua significância estatística, indica a relação de causalidade das variáveis (Enders 2004). E é justamente a análise da relação de causalidade entre receita e despesas governamentais o nosso objetivo final neste trabalho. 


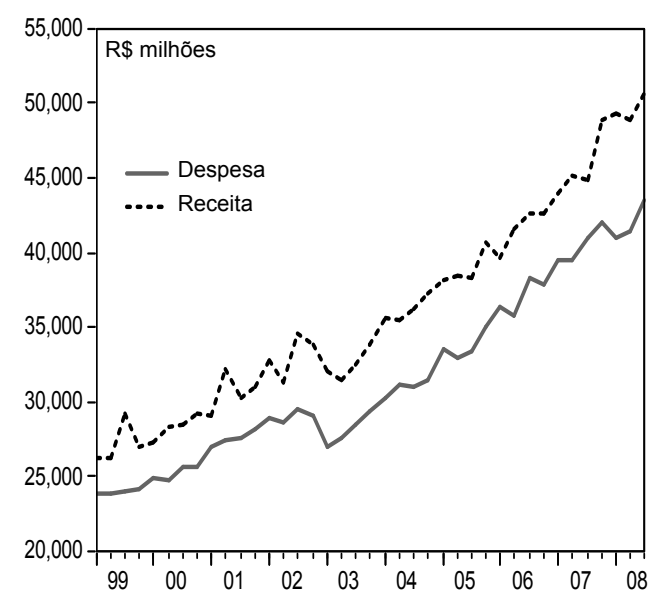

Fonte: Secretaria do Tesouro Nacional

Figura 1: Evolução da receita e despesa primária

\subsection{Dados}

Os testes econométricos envolvem as seguintes variáveis:

Receita: receita primária total líquida a preços constantes e dessazonalizada.

Despesa: despesa primária total a preços constantes e dessazonalizada ${ }^{3}$.

PIB: PIB real dessazonalizado.

A fonte de dados é a Secretaria do Tesouro Nacional e o IBGE e, como já mencionado, a frequência dos dados é trimestral e compreende o período entre o 1 o trimestre de 1999 e o 3o trimestre de 2008. O início da amostra foi escolhido pois marca o estabelecimento de metas de superávit primário para o setor público consolidado. Já o final da amostra foi selecionado para não incorporar os efeitos da crise internacional iniciada em setembro de 2008.

A Figura 1 mostra a estreita relação entre receita e despesa governamentais e é justamente este comportamento sincronizado das variáveis ao longo do tempo uma das motivações de nossa análise.

Algumas ressalvas são necessárias. Primeiramente, seguindo a abordagem de Baghestani \& McNown (1994), Koren \& Stiassny (1998), consideramos o PIB como uma variável macroeconômica de controle. Como argumentado pelos primeiros autores, a utilização das variáveis como proporção do PIB, tal como feito por Bohn (1991), interfere nas conclusões do autor de que os déficits são corrigidos por gasto futuro ou por mudanças na tributação. Mudanças autônomas nos gastos, por exemplo, podem levar a mudanças no PIB que indiretamente causam o ajuste orçamentário das receitas. Desta maneira, para

\footnotetext{
${ }^{3}$ Os dados de Receita e Despesa correspondem ao resultado fiscal do Governo Central (Governo Federal, Banco Central, e INSS), e não o setor público consolidado. As séries foram deflacionadas pelo Índice de Preços ao Consumidor Amplo (IPCA) e dessazonalizados pelo método Census X-12.
} 
considerar os ajustes independentes de receitas e despesas a choques advindos do orçamento, a variável PIB deve entrar no modelo como variável de controle.

Em segundo lugar, nossa análise se restringe à verificação das receitas e despesas primárias, não levando em consideração as despesas referentes aos juros. A razão para esta abordagem se deve ao fato de o superávit primário do governo central não estar inexoravelmente associado ao pagamento de juros, como se poderia imaginar. Do ponto de vista contábil, se há um acréscimo no saldo do caixa único da União, há uma redução em percentual idêntico na Dívida Líquida do Setor Público porque este saldo é abatido da dívida bruta para efeito deste cálculo. De fato, há uma parcela da conta única do Tesouro exclusivamente destinada ao pagamento da dívida pública federal, costumeiramente chamada de "colchão de liquidez". Não obstante, parte considerável do superávit primário está legalmente vinculada a finalidades específicas que não o pagamento da dívida mobiliária.

Quanto à questão da despesa financeira, entendemos que sua inclusão traria consequências negativas ao estudo. O dispêndio com juros é afetado de forma defasada pelas decisões de política monetária, já que parcela significativa da dívida é prefixada ou indexada a índices de inflação. A inclusão desta despesa financeira (que por simetria teria como consequência adicional a inclusão das receitas financeiras) traria um ruído à análise, no mínimo por inflar despesas e receitas em momentos distintos e de forma dissociada da preconizada pela política fiscal. Por fim, o montante de juros a ser pago é sim levado em conta no processo de formulação do orçamento, mas o efeito é pequeno sobre a execução das despesas primárias, já que a fonte principal de recursos para o pagamento da dívida consubstancia-se na receita de capital.

\section{Resultados}

Para determinar a ordem de integração das séries foram aplicados testes de raiz unitária ADF e KPSS. Os resultados estão relatados na Tabela 1 e mostram que, para ambos os testes, não foi possível rejeitar a hipótese nula de raiz unitária das séries ao nível de significância de $5 \%$. Isso é um indicativo que o modelo de correção de erros constitui uma boa solução econométrica em nossa investigação.

Uma vez concluído que as séries são $I(1)$, o próximo passo é definir o número de defasagens do VAR a fim de proceder com a metodologia de cointegração. A Tabela 2 mostra que os Critérios de Informação sugerem um VAR irrestrito com 1 defasagem ${ }^{4}$.

Uma vez decidido o número de defasagens do modelo, segue-se com a estimação do teste de cointegração, com o intuito de testar a existência de uma relação de longo prazo comum entre receita, despesa e PIB. Além disso, deseja-se avaliar qual é a dinâmica de curto prazo entre essas variáveis por meio do coeficiente de ajuste do modelo de correção de erros. Na determinação do modelo de cointegração foi considerado um modelo com constante no vetor de cointegração e tendência linear nos dados. As estatísticas do traço e do máximo autovalor apontam para a existência de um vetor de cointegração. Isso significa que as 3 variáveis possuem uma tendência estocástica comum,

\footnotetext{
${ }^{4}$ Testes de diagnóstico adicionais foram realizados a fim de inferir se a especificação proposta pelos critérios de informação realmente estavam produzindo resíduos do tipo ruído branco.
} 
Tabela 1: Testes de raiz unitária

\begin{tabular}{|c|c|c|c|c|c|c|}
\hline \multicolumn{7}{|c|}{$\mathrm{ADF}$} \\
\hline \multirow[t]{2}{*}{ Séries } & \multirow{2}{*}{$\begin{array}{l}\text { Estatística } \\
\text { de teste }\end{array}$} & \multirow[t]{2}{*}{ Lag } & \multicolumn{2}{|c|}{$\begin{array}{l}\text { Valores } \\
\text { críticos }\end{array}$} & \multicolumn{2}{|c|}{$\begin{array}{c}\text { Rejeita } H_{0} \\
\text { raiz unitária }\end{array}$} \\
\hline & & & $1 \%$ & $5 \%$ & $1 \%$ & $5 \%$ \\
\hline DESPESA & $-1,61$ & 0 & $-4,21$ & $-3,52$ & Não & Não \\
\hline RECEITA & $-1,34$ & 1 & $-4,21$ & $-3,52$ & Não & Não \\
\hline PIB & $-0,07$ & 0 & $-4,21$ & $-3,52$ & Não & Não \\
\hline \multicolumn{7}{|c|}{ KPSS } \\
\hline \multirow[t]{2}{*}{ Séries } & \multirow{2}{*}{$\begin{array}{l}\text { Estatística } \\
\text { de teste }\end{array}$} & \multirow[t]{2}{*}{ Lag } & \multicolumn{2}{|c|}{$\begin{array}{l}\text { Valores } \\
\text { críticos }\end{array}$} & \multicolumn{2}{|c|}{$\begin{array}{c}\text { Rejeita } H_{0} \\
\text { estacionaridade }\end{array}$} \\
\hline & & & $1 \%$ & $5 \%$ & $1 \%$ & $5 \%$ \\
\hline Despesa & 0,17 & 5 & 0,21 & 0,14 & Não & Sim \\
\hline Receita & 0,20 & 4 & 0,21 & 0,14 & Não & Sim \\
\hline PIB & 0,19 & 5 & 0,21 & 0,14 & Não & Sim \\
\hline
\end{tabular}

Nota: Estimações com constante e tendência

Tabela 2: Critério de seleção do melhor modelo

\begin{tabular}{cccc}
\hline Defasagens & AIC & SC & HQ \\
\hline 0 & $-10,19$ & $-10,06$ & $-10,15$ \\
1 & $-15,35^{*}$ & $-14,84^{*}$ & $-15,17^{*}$ \\
2 & $-15,23$ & $-14,34$ & $-14,91$ \\
3 & $-15,05$ & $-13,77$ & $-14,59$ \\
\hline
\end{tabular}

AIC: Critério de Informação AKaike;

SC: Critério de Informação Schwarz;

HQ: Critério de Informação Hnannan-Quinn;

${ }^{*}$ indica a ordem selecionada por cada critério.

isto é, há uma relação de longo prazo entre as séries, ainda que haja desvios de trajetória no curto prazo. Os resultados estão expostos na Tabela 3.

A Tabela 4 reporta os parâmetros estimados do vetor de cointegração detectado. Nota-se que os coeficientes da despesa e do PIB apresentam sinais esperados no vetor de cointegração ${ }^{5}$. A Tabela 4 também reporta a análise da exogeneidade (causalidade no sentido Granger) dentro do contexto do Modelo de Correção de Erros. O objetivo é examinar como se corrigem eventuais erros do equilíbrio de longo prazo. Em outras palavras, dada a relação de longo prazo existente entre as variáveis, o intuito é verificar qual variável se ajusta (dado um choque no sistema) para garantir o equilíbrio de longo prazo já detectado. Os resultados estão na parte inferior da Tabela 4. Nota-se que o coeficiente de ajuste da receita é estatisticamente significante (teste $t=-6.85$ ), ao passo que o coeficiente de ajuste da despesa não possui significância estatística $(t=-0,49)^{6}$. Isso significa que a receita é a variável responsável pelos

\footnotetext{
${ }^{5}$ Não foi possível a partir do teste razão de verossimilhança aceitar a hipótese de que o vetor de cointegração seja igual a $(1,-1,-1)$.

${ }^{6}$ Sob a condição de que há uma relação de cointegração, o teste de razão de verossimilhança
} 
Tabela 3: Teste de Cointegração: Receitas e Despesas Governamentais

\begin{tabular}{lrrrrrrr}
\hline $\begin{array}{l}\text { Há } r \\
\text { vetores } \\
\text { cointegração }\end{array}$ & Autovalor & Traço $\begin{array}{c}\text { Valor } \\
\text { Crítico }\end{array}$ & Prob. $^{* *}$ & Máximo & \multicolumn{1}{c}{$\begin{array}{c}\text { Crítico } \\
(5 \%)\end{array}$} & Prob. $^{* *}$ \\
\hline$r=0^{*}$ & 0,61 & 50,35 & 29,80 & 0,0001 & 37,08 & 21,13 & 0,0001 \\
$r=1$ & 0,24 & 13,27 & 15,49 & 0,1053 & 10,89 & 14,26 & 0,1599 \\
$r=2$ & 0,06 & 2,38 & 3,84 & 0,1228 & 2,38 & 3,84 & 0,1228 \\
\hline${ }^{*}$ denota rejeição de $H_{0}$ com um nível de significância de $5 \%$ \\
${ }^{* *} P$-valores baseados em MacKinnon-Haug-Michelis (1999)
\end{tabular}

Tabela 4: Vetor de cointegração e modelo de correção de erros

\begin{tabular}{lccc}
\hline \multicolumn{1}{c}{ Vetor de Cointegração } & CointEq1 & & \\
\hline RECEITA(-1) & 1,000 & & \\
DESPESA(-1) & $-0,65$ & & \\
& $(0,109)$ & & \\
PIB(-1) & {$[-5.99]$} & & \\
\multicolumn{4}{c}{$-0,71$} \\
& $(0,205)$ & & \\
C & {$[-3,46]$} & & \\
\hline Modelo de Correção de Erros & RECEITA & DESPESA & PIB \\
\hline CointEq1 & $-0,30$ & & \\
& $(0,167$ & $-0,08$ & 0,03 \\
& {$[-6,85]$} & $(0,169)$ & $(0,05)$ \\
C & 0,017 & $0,016]$ & {$[0,58]$} \\
& $(0,004)$ & $(0,004)$ & 0,000 \\
& {$[3,97]$} & {$[3,74]$} & {$[6,10]$} \\
\hline
\end{tabular}

Obs: Desvio Padrão entre parênteses e estatística $t$ entre colchetes.

ajustes que devem ocorrer no curto prazo, dado a incidência de algum choque, siga seu equilíbrio de longo prazo.

Em termos econométricos, a despesa não responde às discrepâncias em relação ao equilíbrio de longo prazo. Portanto, essa variável é caracterizada como exogenamente fraca. Em termos de causalidade, enquanto a receita não Granger-causa a despesa, o contrário pode ser observado, isto é, a despesa Granger-causa a receita.

Desta maneira, pode-se concluir que a hipótese "gastar e arrecadar" adaptase melhor ao caso brasileiro. Isso é um forte indicativo de que elevações temporárias nos dispêndios governamentais provocam mudanças permanentes na arrecadação pública.

\section{Conclusão}

O objetivo deste artigo foi analisar a relação de longo prazo e a causalidade entre a arrecadação governamental brasileira e a despesa pública. Nossa análise enfatizou o período compreendido entre o 1 o trimestre de 1999 e o 3o trimestre de 2008. Neste caso, as análises econométricas mostraram que a

aceita a hipótese nula de que as variáveis DESPESA e PIB sejam fracamente exógenas no longo prazo. 
arrecadação foi a variável de ajuste que garantiu o equilíbrio orçamentário do governo. Desta maneira, o regime fiscal no Brasil pode ser caracterizado como do tipo "gastar e arrecadar", ou seja, o Estado brasileiro pode ser qualificado pela utilização de um modelo fiscal cujas elevações temporárias nos dispêndios governamentais provocam mudanças permanentes na arrecadação pública.

\section{Referências Bibliográficas}

Baffes, J. \& Shah, A. (1994), 'Causality and comovement between taxes and expenditures: Historical evidence from Brazil, and argentina', Journal of Development Economics 44, 311-331.

Baghestani, H. \& McNown, R. (1994), 'Do revenues or expenditures respond to budgetary disequilibria?', Southern Economic Journal 61, 311-322.

Barro, R. (2003), There's a lot to like about bush's tax plan, Technical report, Business Week.

Barro, R. \& Sala-I-Martin, X. (1990), Economic growth and convergence across the united states, Technical report, NBER.

Batolla, F. (2004), Política monetária e sustentabilidade da dívida pública, Master's thesis, Universidade de São Paulo.

Becker, G., Lazear, E. \& Murphy, K. (2003), 'The double benefit of tax cuts', The Wall Street Journal p. A20.

Bohn, H. (1991), 'Budget balance through revenue or spending adjustments? some historical evidence for the united states', Journal of Monetary Economics pp. 333-359.

Chang, T. \& Chiang, G. (2009), 'Revisiting the government revenueexpenditure nexus: Evidence from 15 oecd countries based on the panel data approach', Finance a úver-Czech Journal of Economics and Finance 59.

Chang, T., Lui, W. \& Caudill, S. (2002), 'Tax-and-spend, spend-and-tax, or fiscal synchronization: New evidence for ten countries', Applied Economics 12, 1553-1561.

Cheng, B. (1999), 'Causality between taxes and expenditures: Evidence from latin american countries', Journal of Economics and Finance 23, 184-192.

Darrat, A. F. (1998), 'Tax and spend, or spend and tax? an inquiry into the turkish budgetary process.', Southern Economic Journal 64, 940-956.

Devarajan, S., Swaroop, V. \& Zou, H. (1996), 'The composition of public expenditure and economic growth', Journal of Monetary Economics 37, 313-344.

Enders, W. (2004), Applied econometric time series, Hoboken.

Friedman, M. (1967), Fiscal responsibility, Technical report, Newsweek.

Gamboa, U. \& Silva, R. (2004), Nova evidência sobre a sustentabilidade da política fiscal brasileira: Cointegração, quebras estruturais e senhoriagem, in 'Seminários BACEN-USP de Economia Monetária e Bancária.'. 
Hakkio, C. \& Rush, M. (1991), 'Is the budget deficit too large?', Economic Inquiry 29, 429-445.

Issler, J. \& Piqueira, N. (2000), 'Estimating relative risk aversio, the discount rate, and the intertemporal elasticity of substitution in consumption for Brazil using three types of utility function', Brazilian Review of Econometrics 20, 200-238.

Johansen, S. (1988), 'Statistical analysis of cointegration vectors', Journal of Economical Dynamics and Control 12, 231-254.

Koren, S. \& Stiassny, A. (1998), 'Tax and spend or spend and tax? an international study.', Journal of Policy Modelling 20, 163-191.

Mattos, E. \& Rocha, F. (2001), 'Correção monetária e o equilíbrio do orçamento', Pesquisa e Planejamento Econômico 31.

Mello, L. (2008), 'Estimating a fiscal reaction function: The case of debt sustainability in Brazil.', Applied Economics 40, 271-284.

Meltzer, A. \& Richard, S. (1981), 'A rational theory of the size of government', Journal of Political Economy 89, 914-927.

Musgrave, R. (1966), Principles of budget determination, in H. Cameron \& W. Henderson, eds, 'Public Finance: Selected Readings', Random House, New York.

Pastore, A. C. (1995), 'Por que a política monetária perde eficácia?', Revista Brasileira de Economia 50, 281-311.

Payne, J. (1998), 'The tax-spend debate: Time series evidence from state budgets', Public Choice 95, 3-4.

Peacock, A. \& Wiseman, J. (1961), The growth of public Expenditures in UK, Princeton University Press, Princeton.

Rocha, F. (1997), 'Long-run limits on the brazilian government debt', Revista Brasileira de Economia 51, 210-222.

Romer, C. \& Romer, D. (2007), Starve the beast or explode the deficit? the effects of tax cuts on government spending, Technical report, University of California.

VonFurstenberg, G., Green, R. \& Jeong, J. (1986), 'Tax and spend, or spend and tax?', The Review of Economics and Statistics 68, 179-188. 